\title{
in silico Exploration of Molecular Mechanism and Potency Ranking of Clinically Oriented Drugs for Inhibiting SARS-CoV-2's Main Protease
}

\author{
Tien Huynh, ${ }^{\dagger}$ Haoran Wang, ${ }^{\ddagger}$ and Binquan Luan*, ${ }^{*}$ \\ $\dagger$ †omputational Biological Center, IBM Thomas J. Watson Research, Yorktown Heights, \\ New York 10598, USA \\ $\ddagger$ Neoland Biosciences, Medford, Massachusetts, 02155, USA \\ E-mail: bluan@us.ibm.com
}

\begin{abstract}
Currently, the new coronavirus disease 2019 (COVID-19) is a global pandemic without any well calibrated treatment. To inactivate the SARS-CoV-2 virus that causes COVID19, the main protease (Mpro) that performs key biological functions in the virus has been the focus of extensive studies. With the fast-response experimental efforts, the crystal structures of Mpro of the SARS-CoV-2 virus have just become available recently. Herein, we theoretically investigated the binding mechanism between the Mpro's pocket and various marketed drug molecules being tested in clinics to fight COVID-19 that show promising outcomes. Combining all existing experiment results with our computational ones, we revealed an important ligand-binding mechanism for the Mpro that the binding stability of a ligand inside the Mpro pocket can be significantly improved if the partial ligand occupies the so-called "anchor" site of the Mpro. Along with the
\end{abstract}


high-potent drugs/molecules (such as nelfinavir and curcumin) revealed in this study, the newly discovered binding mechanism paves the way for further optimizations and designs of Mpro's inhibitors with a high binding affinity.

Coronavirus disease 2019 (COVID-19) is a viral respiratory disease of zoonotic origin caused by the novel severe acute respiratory syndrome coronavirus 2 (SARS-CoV-2) virus. COVID-19 first emerged in the city of Wuhan (China) at the end of 2019, but now has turned into a global pandemic reported in all continents just after a few short months. SARS-CoV2 appears to be highly contagious and spreads mainly between human to human through respiratory droplets from coughing and sneezing of the infected persons as well as by fomites. SARS-CoV-2 belongs to a family of viruses named coronaviruses for the crown like spikes on their surface that can infect bats, birds, pigs, cows and other mammals and mutate easily to transfer from animals to humans.${ }^{1}$ Before the COVID-19 outbreak, six strains of such virus already have been identified as human pathogens known to cause viral respiratory illness. However, not all of them are highly pathogenic. For examples, HCoV-229E, HCoVNL63, HCoV-OC43 and HCoV-HKU1 merely cause a common cold. In contrast, both the severe acute respiratory syndrome coronavirus $(\mathrm{SARS}-\mathrm{CoV})^{2}$ and Middle East respiratory syndrome coronavirus (MERS-CoV) ${ }^{3}$ have caused large-scale outbreaks during the past two decades with significant case-fatality rates (9.6\% for SARS and $34 \%$ for MERS). As for COVID-19, its case-fatality rate remains uncertain given the pandemic is still in its early stages.

Currently, it is well known that the SARS-CoV-2's main protease (Mpro) constitutes one of the most attractive antiviral drug targets, because the viral maturation almost exclusively relies on the Mpro's activity. For example, maturation of twelve non-structural proteins (Nsp4-Nsp16), including critical proteins like the RNA-dependent RNA polymerase (RdRp, Nsp12) and helicase (Nsp13), requires the cleavage through the Mpro. It has been demonstrated in experiment that the Mpro inhibition prevented viral replication in multiple studies. ${ }^{4,5}$ Considered as the Achille's heels of SARS-CoV-2, the Mpro is therefore among the 
top candidates for drug discovery. Additionally, the Mpro's inhibitor(s) is likely to inactivate virus in different cell types in different organs, independent of the various receptors/hostproteases (on the cell membrane) required for virus entry.

So far, a specific Mpro inhibitor is still missing for the SARS-CoV-2 virus. Irreversible inhibitors like N3 are efficacious and have been proven to inhibit SARS-CoV-2 virus in in vitro viral proliferation models with moderate efficacy (EC50: 4-5 $\mu \mathrm{M}) .{ }^{5}$ However, development of these tool drugs into an approved drug could take years to accomplish. In an BioRxiv preprint, ${ }^{6}$ a few marketed drug such as Ebselen, Disulfiram, Tideglusib, and Carmofur have exhibited EC50 of $0.67 \mu \mathrm{M}, 9.35 \mu \mathrm{M}, 1.55 \mu \mathrm{M}$ and $1.82 \mu \mathrm{M}$ respectively with an in vitro enzymatic assay, which translate to EC50 of $4.6 \mu \mathrm{M}$ in antiviral activity for Ebselen (best in class), comparing to EC50 of $16.77 \mu \mathrm{M}$ for N3. ${ }^{5}$ These experiments validated that Mpro could be a viable anti-viral target, albeit additional efforts are needed to search for more potent and specific anti-viral drugs with a better safety margin than Ebselen that is an (irreversible) inhibitor for the Mpro and many other enzymes in a broad spectrum of tissues with significant cellular toxicity. ${ }^{7}$ Motivated by the fact that Mpro can be inhibited by multiple drug-like ligands, we conjectured that a range of drug molecules may efficaciously interact with the Mpro pocket. Given the urgency, we used in silico methods to explore a set of $(\sim 20)$ marketed drugs that have exhibited high promises in clinics, aiming to identify the potential high-potent ones for the Mpro inhibition and discover a common binding mechanism for these drug molecules inside the Mpro's pocket.

Understanding the structural determinants for protein-ligand complex at the atomic level is crucial for designing ligands with high specificity and affinity to a target protein. Moreover, gaining insight into the mechanisms responsible for the protein-ligand recognition and binding greatly facilitates the discovery and development of drugs for treatment of the underlying disease. We carried out all-atom molecular dynamics (MD) simulations that are widely used in the studies of biomolecules, ${ }^{8,9}$ guided with fast and efficient docking studies. Besides the identification of several high-potent drugs/molecules, we unveiled the consensus 
binding mechanism that a ligand prefers to bind the "anchor" site of the Mpro pocket, which might facilitate the future design and optimization of an inhibitor for the SARS-CoV-2's Mpro.

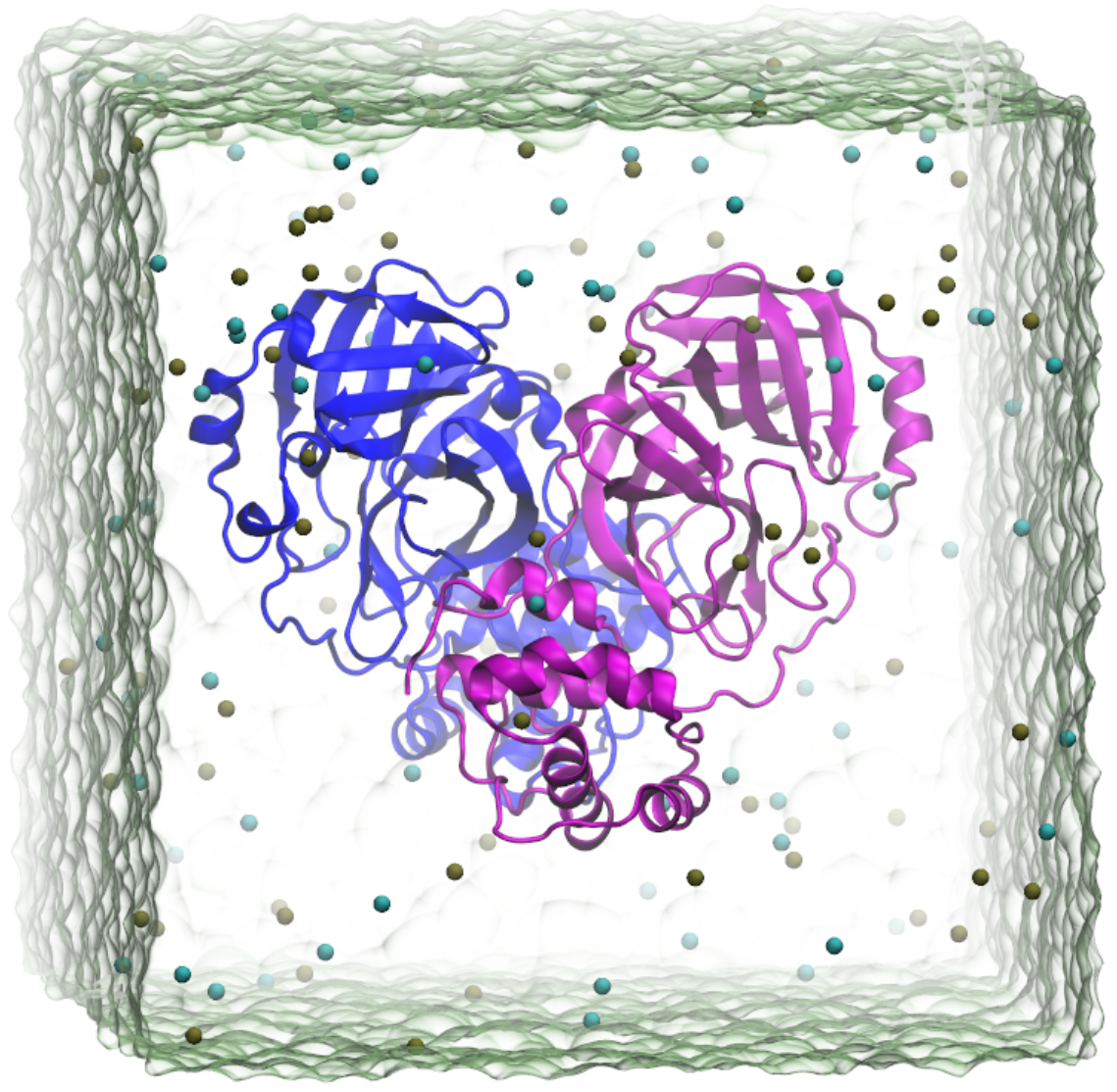

Figure 1: The MD simulation system for the SARS-CoV-2's Mpro. Two monomers in the Mpro dimer (PDB: 6LU7) are in cartoon representation and colored in blue and purple respectively. $\mathrm{K}^{+}$and $\mathrm{Cl}^{-}$ions are shown as van der Waals spheres and are colored in tan and cyan respectively. Water are shown transparently.

In our in silico studies, we used the $\mathrm{NAMD}^{10}$ package for studying the structures of the stand along apo Mpro as well as the ligand-bound one. We first equilibrated the crystal structure of the SARS-CoV-2 Mpro (PDB: 6LU7) in the physiologically relevant environment. The simulation system is illustrated in Fig. 1. The dimer structure of the Mpro (colored in blue and purple respectively) is illustrated in the cartoon representation. The entire protein was then solvated in a water box measures $97.4 \times 97.4 \times 97.4 \AA^{3} .88 \mathrm{~K}^{+}$and 
$80 \mathrm{Cl}^{-}$ions were added into the solution to neutralize the net charge of the protein and set the ion concentration to be $0.15 \mathrm{M}$. The MD simulations were carried out on the IBM Power-cluster. We applied the TIP3P model ${ }^{11,12}$ for water, standard ion force field ${ }^{13}$ and the CHARMM36 force field ${ }^{14}$ for the protein. A smooth cutoff (10-12 $\AA$ ) was used to calculate van der Waals energies. Electrostatic interactions were calculated using the particle-mesh Ewald (PME) method (grid size $\sim 1 \AA$ ). With the SETTLE algorithm ${ }^{15}$ enabled to keep all bonds rigid, the simulation time-step was 2 fs

The entire simulated system was first equilibrated at 1 bar and $300 \mathrm{~K}$, with all backbone atoms in the Mpro harmonically restrained (spring constant $k=1 \mathrm{kcal} / \mathrm{mol} / \AA^{2}$ ). In the subsequent production simulation, the restraint was removed and the Mpro was further equilibrated in the NPT ensemble. The Langevin dynamics was applied to maintain the constant temperature $(300 \mathrm{~K})$ in the simulated system and the pressure was kept constant at 1 bar using the Nosé-Hoover method. ${ }^{16}$

During the 125 ns production run, the overall dimer structure was stable and the root mean square deviations (RMSD) of the protein's backbone calculated against the crystal (initial) one (PDB: 6LU7) saturated at $1.7 \AA$ after about $10 \mathrm{~ns}$. Note that in the crystal structure there exists the N3 ligand (inside the Mpro's pocket) that is covalently linked to the Mpro, i.e. the irreversible binding. It is conceivable that the Mpro' pocket may slightly change its conformation once the N3 molecule is removed. When we are close to finish writing this paper, the apo structure (PDB:6Y2E) of the Mpro became available in the protein data bank. The RMSDs calculated against this latest Mpro's crystal structure without any bound ligand shows a smaller mean value, indicating that without a bound ligand the equilibrated protein structure is closer to the apo structure.

Interestingly, as shown in the inset of Fig. 2a, we found that during the equilibration, the positively charged N-terminus SER1 in one monomer (of the Mpro) and the negatively charged GLU166 in the other monomer formed a stable salt-bridge in the electrolyte. This observation is particularly important as the Mpro's pocket (shown as a shaded oval in the 

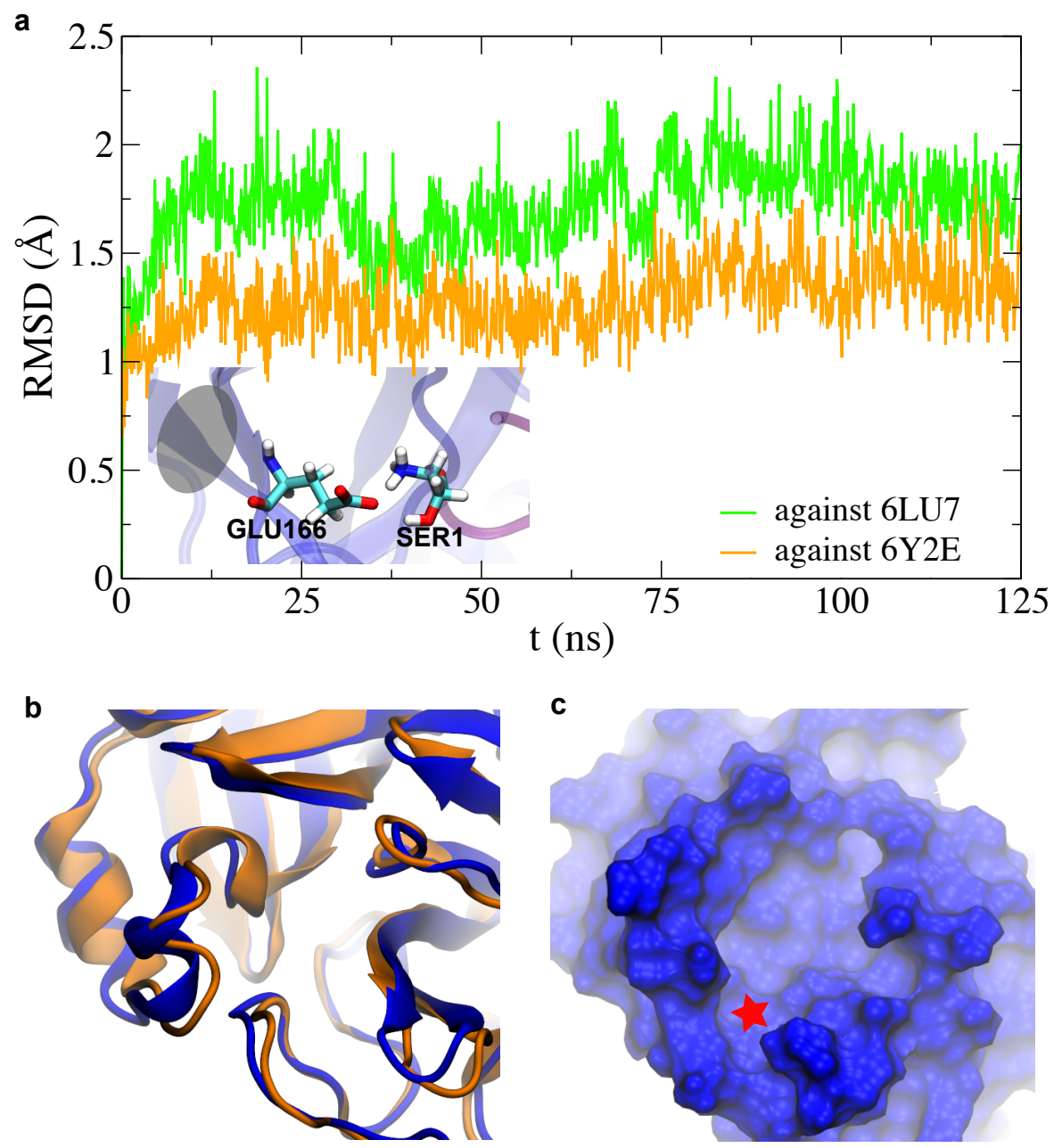

Figure 2: Molecular dynamics simulation of the SARS-CoV-2's Mpro. a) Root mean square deviations (RMSD) of simulated protein structure against the crystal (initial) structure (PDB: 6LU7) and against the crystal structure (PDB: 6Y2E). The inset illustrates a saltbridge formed by the negatively charged GLU166 of one moner (blue) and the positively charged N-terminus SER1 of the other monomer (purple) during the MD equilibration; the gray oval shows where the Mpro pocket is. b) The cartoon representations of the equilibrated (blue) and the aligned crystal Mpro's pockets without the bound N3 ligand. c) The molecular surface representation of the equilibrated Mpro's pocket. 
inset of Fig. 2a) is right beside the GLU166, indicating that this salt-bridge plays a critical role to the pocket's stability. In Fig. 2b, we show some small structural differences between the equilibrated Mpro's pocket (blue) and the aligned apo structure (orange), which signifies the importance of using the Mpro's structure after being equilibrated in the MD simulation for further docking studies. Using the molecular surface representation, we highlight the ligand-binding pocket of the equilibrated Mpro in Fig. 2c.

For predicting the binding mode and affinity of a ligand relative to a protein, Over the last few decades, there are many computer aided tools and programs developed for both commercial and academic uses such as Glid, ${ }^{17}$ GOLD,${ }^{18}$ MOE DOCK, ${ }^{19}$ rDOCK, ${ }^{20}$ AutoDock Vina, ${ }^{21}$ to name a few. In this study, for the docking part, we employed AutoDock Vina which is a successor of the most cited docking software, AutoDock, ${ }^{22}$ with significant improvement in terms of accuracy and performance. Indeed, it has been shown that the scoring power of AutoDock Vina is competitive to the commercial ones despite it is an open-source program. ${ }^{23}$

We carried out the docking calculations on an IBM power node with 24 physical cores and 192 CPUs. For preparation of the ligands and target protein to be applied in AutoDock Vina, we used the scripts prepare-ligand4.py and prepare-receptor4.py provided with the AutoDock Tools[6,7] suite to generate the corresponding input files in PDBQT format which extends the PDB format with additional fields of partial charge and atom type. In order to increase the chance of finding the minimum binding energy to predict where and how a putative ligand can best bind the target protein, we set the exhaustiveness parameter of the program to 100 for each search.

Figure 3a shows the Mpro protein (equilibrated) with a ligand-binding pocket, surrounded by a rectangular box within which a ligand is docked. We first used the available crystal structure (PDB: 6Y2F) with a bound O6K molecule (shown in Fig. 3b) as a reference to calibrate the docking procedures. After searching hundreds of poses, the program yielded the best pose (shown in Fig. 3c) very similar to the one in the crystal structure (shown in 
Fig. 3d). The obtained docking (affinity) score using the highly-optimized score-function in Autodock Vina is $-7.4 \mathrm{kcal} / \mathrm{mol}$. The main difference is present in the O6K's Boc group (containing the -C- $\left(\mathrm{CH}_{3}\right)_{3}$ group). In the equilibrated structure (Fig. 3c), the tail resides inside the pocket region between the beta sheets (residues from TYR161 to ASP176) and the coil (residues from GLY183 to ALA194). Hereafter, we refer this special pocket region (also labeled with a star in Fig. 2c) as the "anchor" site. However, in the crystal structure, the same Boc group is present above the beta sheet, which is likely resulted from the competitive occupancy of the "anchor" site by an dimethyl sulfoxide (DMSO) molecule (Fig. 3d).

a

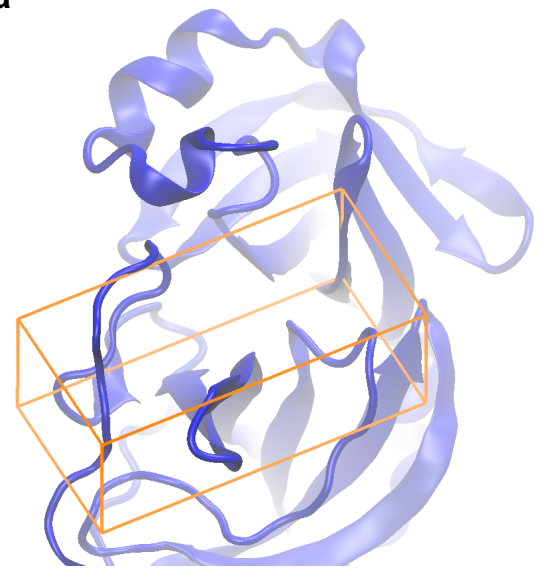

C

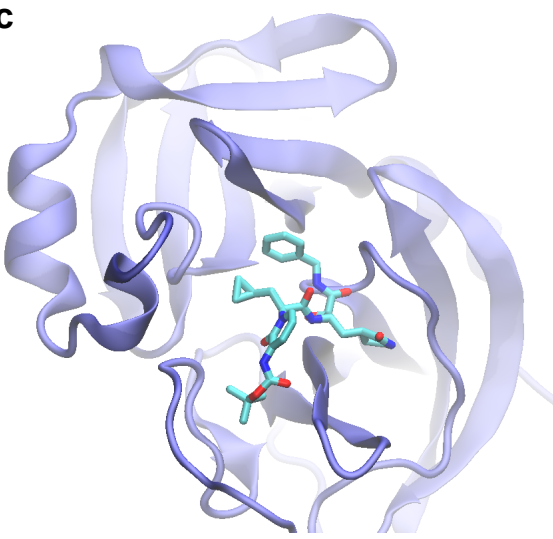

b

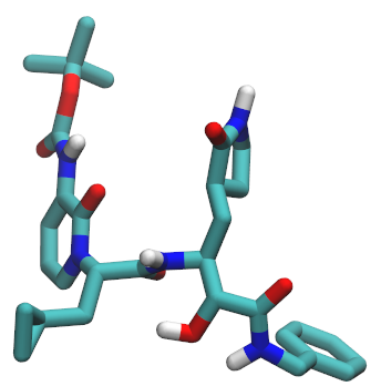

d

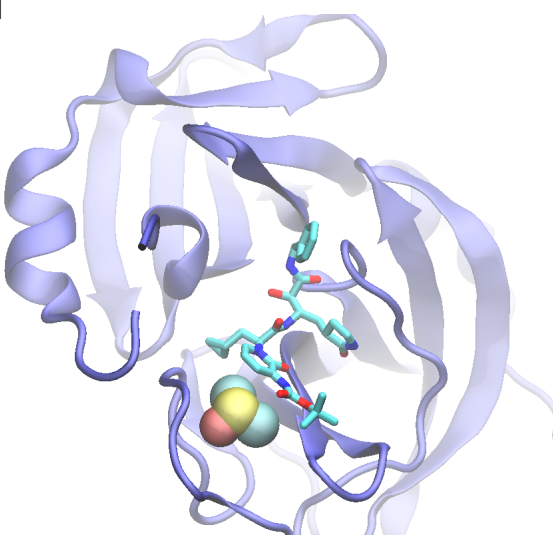

Figure 3: Docking study of the O6K molecule in the pocket of the Mpro. a) An illustration of the Mpro's pocket with a rectangular box within which a drug molecule is docked. b) A stick representation of the O6K molecule. c) The best docked pose of the O6K molecule in the Mpro's pocket. d) The crystal structure (PDB: 6Y2F) of the SARS-CoV-2's main protease with the O6K molecule and a cocrystallization agent dimethyl sulfoxide. 
Following the same docking protocol, we evaluated about 20 drugs (or compounds), most of which are currently tested in clinics for the COVID-19 disease. For these promising drugs, their antiviral mechanisms are still illusive. Given the importance of Mpro, we investigate whether the Mpro inhibition may be a part of the mechanism of action. Besides the O6K molecule shown in Fig. 3b, Fig. 4(a-r) respectively illustrate molecular structures of chloroquine, bromhexine, favipiravir, dipyridamole, ambroxol, hydroxychloroquine, montelukast, cinaserin, GS-441524, kaempferol, lopinavir, entecavir, umifenovir, quercetin, remdesivir, nelfinavir, curcumin and N3. Here N3 along with O6K were used in the docking studies as controls. Consistent with their presence inside the Mpro's pocket in the crystal environment, we found that two best docking scores belong to N3 $(-7.1 \mathrm{kcal} / \mathrm{mol})$ and $\mathrm{O} 6 \mathrm{~K}(-7.4 \mathrm{kcal} / \mathrm{mol})$.

Remdesivir is a potent anti-viral drug with EC50 of $0.77 \mathrm{uM}$ towards the SARS-CoV-2 virus in vitro. ${ }^{24}$ It was also reported to be efficacious in a few critically-illed patients. ${ }^{25}$ Remdesivir is a pro-drug which will hydrolyze in vivo. We found that both remdesivir and its metabolite (GS-441524) could complex well with the Mpro with the affinity scores -7.0 and $-6.4 \mathrm{kcal} / \mathrm{mol}$ respectively, (Fig. 4t) which could provide synergistic effects in addition to its RNA dependent RNA polymerase (RdRp) antagonism effects. The score of remdesivir is among the highest category, making it a good candidate for the first line anti-COVID-19 drug. Favipiravir is also a RdRp inhibitor developed by Fujifilm Corp. in Japan, however this molecule is small and didn't show significant binding with the Mpro in our model (Fig. $4 \mathrm{t})$.

Entecavir is another potential inhibitor for RdRp, and had been used for treating hepatitis B virus (HBV) for years with good efficacy and safety profile. With a similar structure to remdesivir's metabolite (GS-441524), entecavir is affordable and widely available, and could be a good alternative as potential inhibitors for both RdRp and Mpro. Fig. 4t shows that the affinity score for entecavir is $-6.4 \mathrm{kcal} / \mathrm{mol}$ that is same as the one for GS-441524. Given by its availability and promising docking score, we further carried out MD simulations to reassure the stable binding of entecavir to the Mpro's pocket (see below). 
a
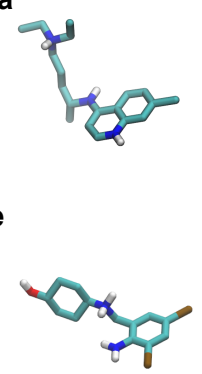

i

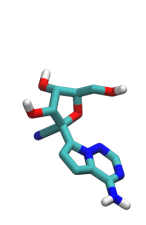

m

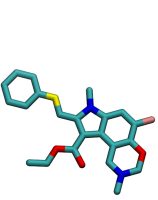

j

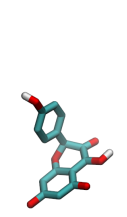

n
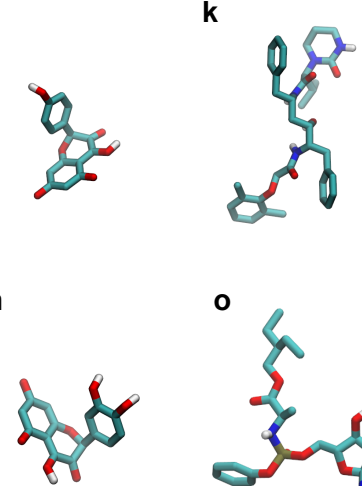

k

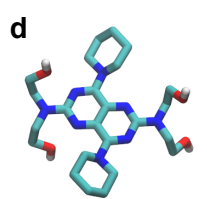

h

g

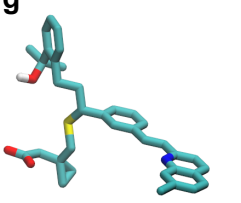

$\ln _{0}$

I

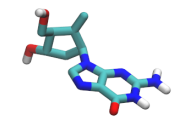

q

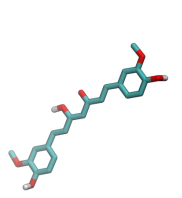

$\mathbf{r}$
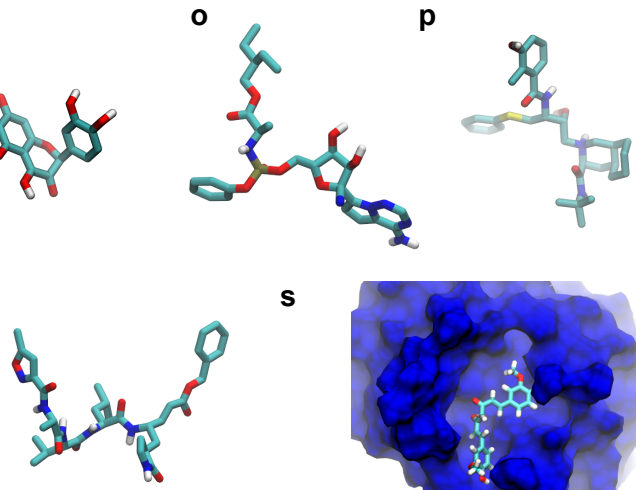

S
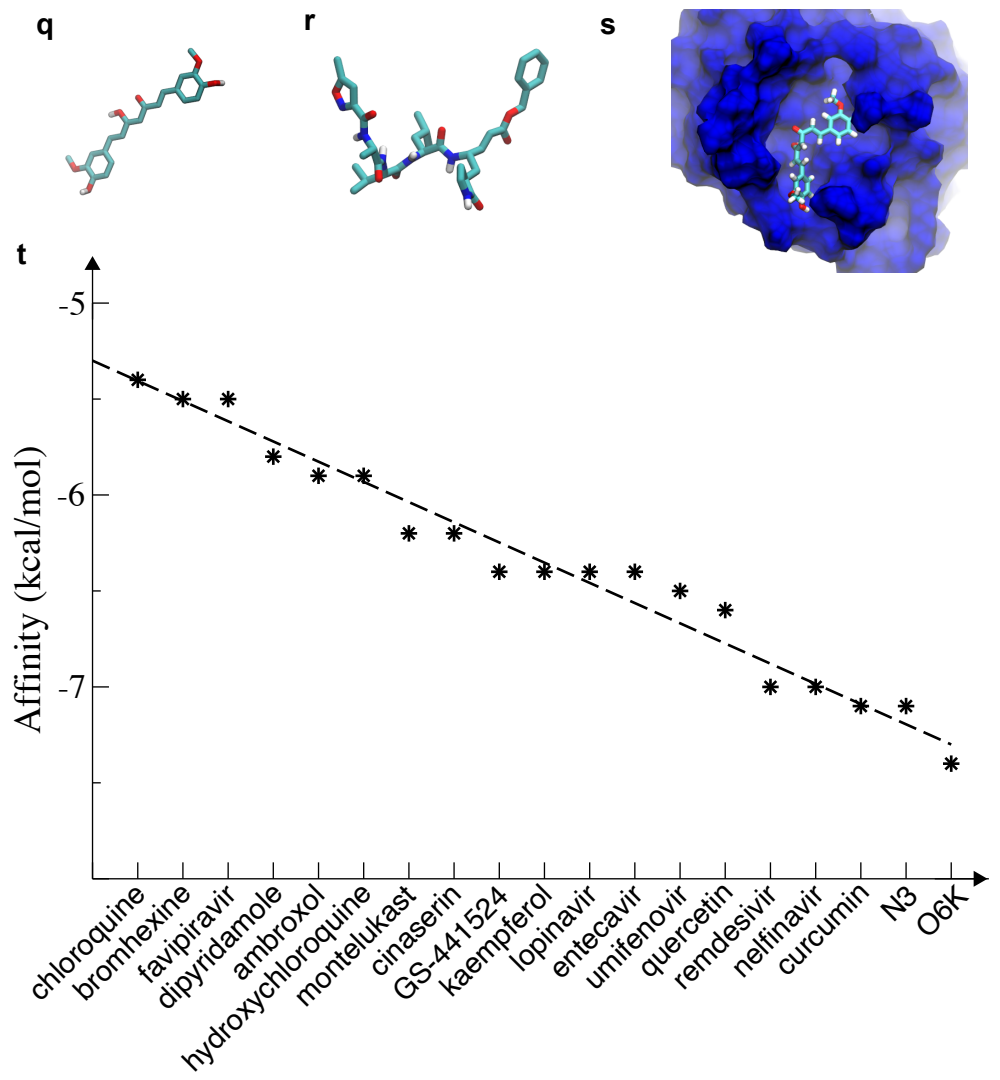

Figure 4: Docking studies of various clinically tried drugs in the pocket of the Mpro. a) chloroquine. b) bromhexine. c) favipiravir. d) dipyridamole. e) ambroxol. f) hydroxychloroquine. g) montelukast. h) cinaserin. i) GS-441524. j) kaempferol. k) lopinavir. l) entecavir. m) umifenovir. n) quercetin. o) rełpdesivir. p) nelfinavir. q) curcumin. r) N3. s) The top pose of curcumin in the Mpro pocket. t) Binding affinities of tested drug molecules docked in the Mpro pocket. 
Curcumin has multifaceted function in curbing inflammation including, IL6, TNF- $\alpha$, IL$1 \beta$ etc. and was also known to the protection over liver and gastrointestinal (GI) tract damage, which is common in COVID-19 pathological condition. ${ }^{26}$ To our surprise, we found curcumin, a widely available food supplement, forms the most stable $(-7.1 \mathrm{kcal} / \mathrm{mol})$ complex with SARS-CoV-2's Mpro among the tested drugs. Its affinity score is as good as the one for N3. Fig. 4t shows the top pose of a curcumin molecule nicely fit inside the Mpro pocket. It is well known that curcumin had a low bioavailability (1\%) which could hamper the utility of its treating systemetic viral infection, therefore it is important to find a viable formula which can deliver sufficient curcumin to the target organ. ${ }^{27}$

However, there are some evidences that orally administered curcumin accumulates in sufficient quantity in the GI tract and liver. Besides the lung, the SARS-CoV-2 virus also infects the GI tract, causing the patients to experience also diarrhea. ${ }^{28,29}$ GI track and liver are immune privileged and may provide a shelter for SARS-CoV-2 virus from attacks by the immune system. ${ }^{30,31}$ Virus from the GI tract would be shedding off even after the lung tissue is free of viral infection, which could pose a great threat to infect others in shared bathrooms or via the aerosol formed in sewage system. Additionally, the GI system also expresses a high level of ACE2 receptors and TMPRSS2, which is critical for the SARS-CoV-2 infection. To prevent the GI system from providing a protective shelter for the SARS-CoV-2 virus, it is beneficial to take drugs (such as curcumin) to curb the virus infection in the GI system during the drug treatment regime, and even after the viral infection being tampered in the lung.

We also found quercetin and kaempherol, natural products enriched in fruits and vegetables, are capable of complexing the Mpro with decent binding affinities, $-6.6 \mathrm{kcal} / \mathrm{mol}$ and $-6.4 \mathrm{kcal} / \mathrm{mol}$ respectively (Fig. 4t). The bioavailability of quercetin supplements is about $24 \%$, comparing to $52 \%$ from isoquercetin-rich onions. ${ }^{32}$ The bioavailability of kaempherol is about $2 \%$, many of which were metabolized in the liver. Note that both quercetin's and kaempherol's bioavailability are better than that of curcumin. 
Nelfinavir is an antiretroviral drug used in the treatment of the human immunodeficiency virus (HIV). Nelfinavir belongs to the class of drugs known as protease inhibitors (PIs) and like other PIs it is almost always used in combination with other antiretroviral drugs. Previously, nelfinavir has been shown to inhibit the replication of SARS-CoV. ${ }^{33}$ Consistently, we found that the affinity score is $-7.0 \mathrm{kcal} / \mathrm{mol}$, placing it among the top candidates (Fig. 4t). So far, nelfinavir has not been tested clinically for treating COVID-19 disease, however our data strongly suggest it as a good candidate with the possible Mpro inhibition. As another HIV PI, lopinavir has a lower affinity score $(-6.4 \mathrm{kcal} / \mathrm{mol}$, Fig. 4t) than the one for nelfinavir. According to the recent clinical trial, ${ }^{34}$ lopinavir worked only modestly in the early phase of infection of SARS-CoV-2 and it is also not strong enough to support significant efficacy at the later stage of viral infection.

Umifenovir or arbidol is an anti-viral drug available in Russian and China, and had been used clinically in treating COVID-19 in China. Umifenovir inhibits SARS-CoV-2 in vitro with a reported IC50 of 30uM. ${ }^{35}$ It has also demonstrated positive results as a post-exposure prophylaxis (PEP) of COVID-19 transmission. ${ }^{36}$ We obtained the moderate Mpro binding in our model with a score of $-6.5 \mathrm{kCal} / \mathrm{Mol}$ (Fig. 4t).

Montelukast is a leukotriene inhibitor used to treat allergies and prevent asthma attacks. It was reported anecdotally to inhibit the Mpro and we validated its binding with the Mpro with a moderate score of $-6.2 \mathrm{kcal} / \mathrm{mol}$ (Fig. 4t). Montelukast could offer other anti-inflammation benefits other than the potential Mpro inhibition.

Bromhexine ${ }^{37}$ and ambroxol ${ }^{38}$ are OTC expectorant drugs and are used in assisting the treatment of COVID-19 in China. We found that they may exhibit weak Mpro antagonism effects according to docking affinities (Fig. 4t). Nevertheless, expectorant drugs normally are highly enriched in lung tissues, which can promote the inhibition of Mpro's activities.

Chloroquine was found to inhibit SARS-CoV-2 in vitro ${ }^{29}$ in clinical tests. Despite very similar chemical properties, the efficacy of hydroxychloroquine (HCQ) is 7 times stronger than that of chloroquine(CQ) in vitro (EC50 $0.7 \mu \mathrm{M}$ v.s $5.4 \mu \mathrm{M}$ in Vero cell infection 
model).$^{39}$ The potency discrepancy calls for other anti-viral mechanism besides the existing hypothesis of $\mathrm{HCQ} / \mathrm{CQ}$ function by neutralizing endosome $\mathrm{pH}$ and inhibiting cathepsin $\mathrm{L},{ }^{40}$ which would predict similar efficacy if not the same, with such minor structural differences between HCQ and CQ.

In support of other unknown targets by $\mathrm{HCQ}$ and $\mathrm{CQ}$, the presence of extracellular proteases like TMPRSS2 and Furin facilitate efficient virus entry on the plasma membrane directly and bypass on the endocytosis pathway. ${ }^{41}$ The fact that HCQ/CQ still poses strong inhibition of virus clinically also suggests $\mathrm{HCQ} / \mathrm{CQ}$ inhibited intracellular common pathway such as the Mpro. Meanwhile, HCQ/CQ can accumulate in the lung with 200-700 fold higher concentration than that of the serum, ${ }^{42}$ which may suffice for Mpro inhibition with sub-optimal affinity score of $-5.9 \mathrm{kCal} / \mathrm{Mol}$ for $\mathrm{HCQ}$ and $-5.0 \mathrm{kCal} / \mathrm{Mol}$ for $\mathrm{CQ}$ (see Fig. 4t).

Dipyridamole exhibits a broad spectrum of anti-viral activities and has been used as an effective anti-COVID-19 drug clinically. ${ }^{43}$ The anti-viral mechanism of dipyridamole involves the inhibition of the Mpro as determined by the surface plasmon resonance (SPR) assay in vitro. ${ }^{43}$ However, the affinity sore is $-5.8 \mathrm{kcal} / \mathrm{mol}$ (Fig. 4t). Additionally, cinanserin was predicted to inhibit the Mpro of both SARS-CoV and SARS-CoV-2, however, we found its binding affinity with Mpro is not very high, in line with a $120 \mu \mathrm{M}$ IC50 for the viral inhibition. ${ }^{6}$ The analog of cinanserin (cmpd-26) perform much better in wet-lab experiments with IC50 of $1.06 \mu \mathrm{M}$ for SARS-CoV Mpro protease, ${ }^{?}$ however, the score of cmpd-26 is not much improved for the SARS-CoV-2 Mpro. These discrepancies may be due to the rough estimations of the binding affinity from the docking study, which suggests the need for a more accurate model such as the MD one that includes the impact of water (e.g. desolvation energy).

To verify the docking results, as an example, we further performed the MD simulation to investigate the stability of entecavir's pose with the best affinity score inside the Mpro pocket (Fig. 5a). The force field parameters for entecavir were obtained from SwissParam. ${ }^{44}$ We applied the same MD simulation protocol used for the apo Mpro equilibration (Fig. 1 

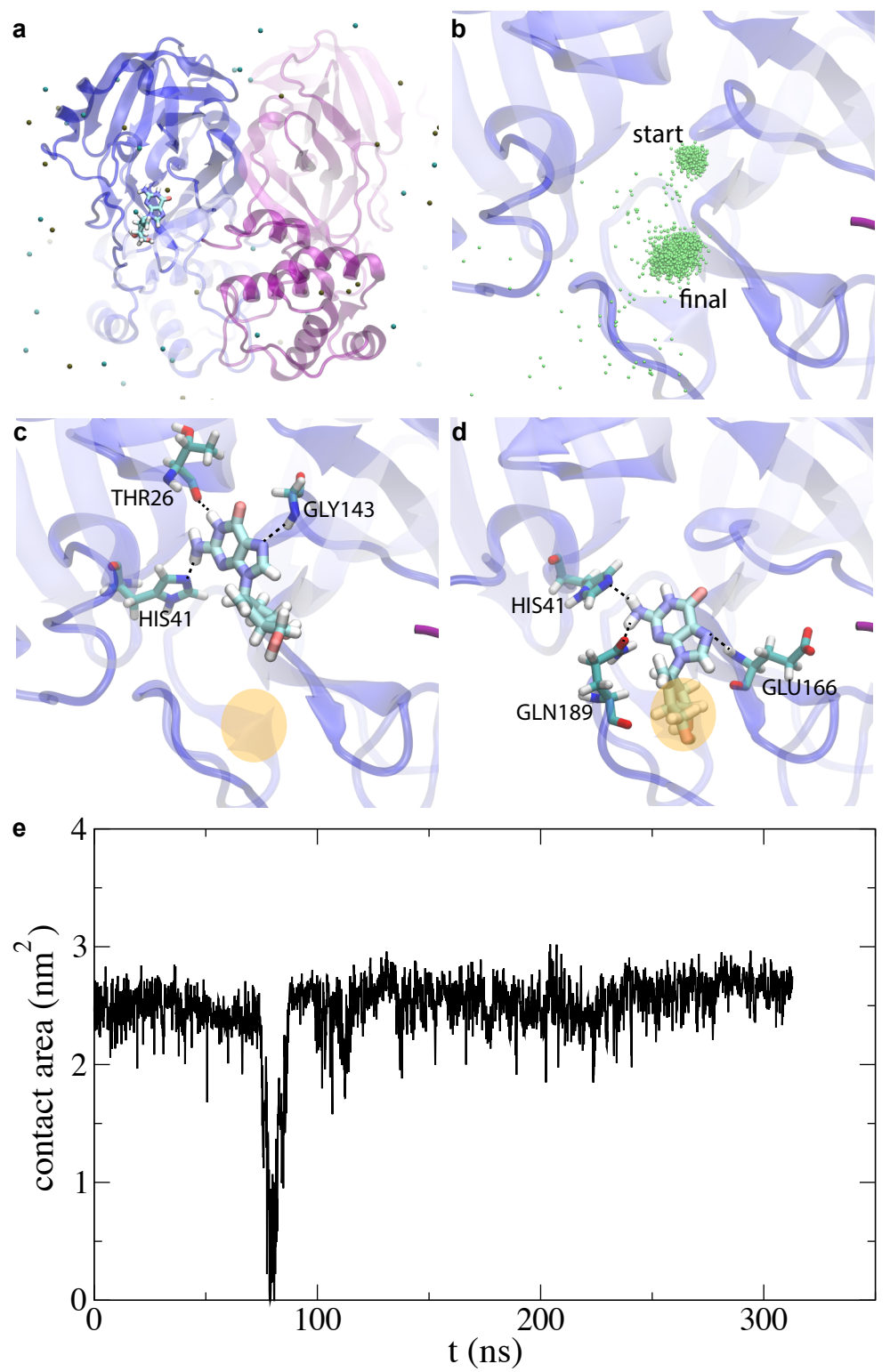

Figure 5: Molecular dynamics simulation of entecavir in the Mpro pocket. a) An enlarged view of the MD simulation system for the Mpro with a bound entecavir molecule. Water is not shown for clarity. b) Centers of mass of the entecavir molecule during the entire MD simulation. c) The entecavir's pose at the beginning of the MD simulation, obtained from the docking study. d) The entecavir's pose after the MD equilibration. e) The time dependency of the contact area between the enticavir molecule and the Mpro pocket. 
and 2). To highlight the positions of the entecavir molecule inside the Mpro pocket, we overlapped the center of mass (COM) of entecavir during the entire MD simulation in Fig. 5b. Notably, there are two clusters of COMs, representing the start and final locations of the entecavir molecule. The entire process for entecavir's repositioning its pose inside the Mpro pocket is illustrated in the movie in the supplementary materials.

Fig. 5c shows the initial pose of the entecavir molecule in the MD simulation, that is also the best pose from the docking study. Inside the Mpro pocket, the entecavir molecule formed hydrogen bonds with the residues THR26, HIS41 and GLY143. Overall, the entecavir's size is smaller than the one for the Mpro's pocket, and the above mention "anchor" site (the orange oval in Fig. 5c) is not occupied. After entecavir's drifting to the new location inside the Mpro, it coordinates with HIS41, GLN189 and GLU166 through hydrogen bonds. Interestingly, the five-membered ring in the entecavir molecule entered the "anchor" site (see orange oval in Fig. 5d), driven by the hydrophobic interaction. Note that the new pose of entecavir found in MD simulation is similar to the eighth pose with a little larger score (-5.9 $\mathrm{kcal} / \mathrm{mol}$ ) discovered in the docking study. This highlights that when including the solvent effect the affinity scores listed in Fig. 4t can be different $(\sim 10 \%)$, which can somewhat affect the rank order.

To further quantitatively demonstrate the dynamic drifting process of entecavir, we calculated the time dependent contact area between the entecavir molecule and the Mpro pocket. Based on the concept of the solvent-accessible surface area, we calculated the surface areas of the Mpro pocket $\left(S_{M}\right)$, the entecavir molecule $\left(S_{E}\right)$ and their complex $\left(S_{T}\right)$. Thus, we define the contact area between the entecavir molecule and the Mpro pocket as $\left(S_{M}+S_{E}-S_{T}\right) / 2$. Fig. 5e shows the calculated contact area during the more than 300 ns MD simulation. In the first about $75 \mathrm{~ns}$, the entecavir molecule stayed in its initial location with an average contact area $\sim 2.4 \mathrm{~nm}^{2}$. After that, it drifted away from its initial location and move into water above the Mpro pocket. The contact area decreased to about zero. Without drifting further into water, the entecavir molecule reentered the Mpro pocket and the contact 
area increased to about $2.6 \mathrm{~nm}^{2}$. During the rest of the MD simulation (about $250 \mathrm{~ns}$ ), the entecavir moleule remained stably in the new location.

Through both the docking and MD studies of about 20 drug molecules, we found that the "anchor" site in the Mpro pocket plays an important role in stabilizing a bound ligand. For example, in the docking study, one end of the curcumin molecule was seen to insert inside the "anchor" site (Fig. 4s). Likewise, in the MD simulation of the entecavir in the Mpro pocket, the entecavir molecule found a more stable binding pose with its five-membered ring inside the "anchor" site. In the crystal structure (PDB: 6LU7), the $-\mathrm{CH}_{3}$ group containing tail of the N3 molecule (Fig. 4r) also occupies the "anchor" site of the Mpro, corroborating the importance of the "anchor" site for the ligand binding.

In the recent experimental study ${ }^{5}$ it was found that the bulky Boc group in the O6K molecule (Fig. 3b) is essential for its binding to the Mpro pocket, without which the ligand becomes inactive. As shown in Fig. 3c, our docking result verifies that the Boc group indeed occupies the "anchor" site, which again emphasizes the importance of the "anchor" site in the ligand's binding with the Mpro pocket.

Additionally, we point out that in the crystal structure (PDB: 6Y2F), there is a DMSO molecule binding inside the "anchor" center (Fig. 3d), which may function to impede the binding of potential inhibitors. It is well known that DMSO was used widely to dissolve compound for biological assays. For example, dipyridamole had low solubility and it inhibited Mpro in a SPR assay with $34 \mu \mathrm{M}$ binding affinity. However, the in vitro viral-cell-based assay showed that the EC50 of dipyridamole can be as low as $0.1 \mu \mathrm{M}$. The SPR assay utilized purified Mpro proteins exposed directly to a compound solution containing $0.5 \%$ of DMSO; the later could compete with dipyridamole for binding with Mpro and results in false negative results.

In summary, we have used in silico methods including the docking and MD simulation to investigate the stability of various ligands bound inside the Mpro pocket. Besides the binding energy, we have also considered comprehensively about the bioavailability and half 
life of the drugs, which translates to effective drug concentration around the targets. We found that several molecules (such as nelfinavir and curcumin) have their docking affinity scores very close to the ligands found experimentally (N3 and O6K). By examining almost 100 different poses of each docked molecule, we realized the importance of the "anchor" site in the Mpro pocket. Besides the hint from docking studies, we confirmed in MD simulation that by occupying the "anchor" site, a ligand can reside inside the Mpro pocket more stably via the hydrophobic interactions. More importantly, the binding mechanism revealed in this work is supported by evidences observed in experimental studies. Therefore, we expect that these in silico results and predictions could shed light on the future discovery of high-potent inhibitors targeting the SARS-CoV-2's Mpro.

\section{Competing Interests}

T. H., H. W. and B. L. declare no competing interests.

\section{Acknowledgement}

B. L. gratefully acknowledges the computing resource and the financial support from the IBM Bluegene Science Program.

\section{Supporting Information Available}

The movie from the trajectory of MD simulation for the entecavir-Mpro complex.

\section{References}

1. Cui, J.; Li, F.; Shi, Z.-L. Origin and evolution of pathogenic coronaviruses. Nature reviews Microbiology 2019, 17, 181-192. 
2. Kuiken, T.; Fouchier, R. A.; Schutten, M.; Rimmelzwaan, G. F.; Van Amerongen, G.; van Riel, D.; Laman, J. D.; de Jong, T.; van Doornum, G.; Lim, W. et al. Newly discovered coronavirus as the primary cause of severe acute respiratory syndrome. The Lancet 2003, 362, 263-270.

3. Zaki, A. M.; Van Boheemen, S.; Bestebroer, T. M.; Osterhaus, A. D.; Fouchier, R. A. Isolation of a novel coronavirus from a man with pneumonia in Saudi Arabia. New England Journal of Medicine 2012, 367, 1814-1820.

4. Anand, K.; Ziebuhr, J.; Wadhwani, P.; Mesters, J. R.; Hilgenfeld, R. Coronavirus main proteinase (3CLpro) structure: basis for design of anti-SARS drugs. Science 2003, 300, $1763-1767$.

5. Zhang, L.; Lin, D.; Sun, X.; Curth, U.; Drosten, C.; Sauerhering, L.; Becker, S.; Rox, K.; Hilgenfeld, R. Crystal structure of SARS-CoV-2 main protease provides a basis for design of improved $\alpha$-ketoamide inhibitors. Science 2020,

6. Jin, Z.; Du, X.; Xu, Y.; Deng, Y.; Liu, M.; Zhao, Y.; Zhang, B.; Li, X.; Zhang, L.; Peng, C. et al. Structure of Mpro from COVID-19 virus and discovery of its inhibitors. bioRxiv 2020,

7. Azad, G. K.; Tomar, R. S. Ebselen, a promising antioxidant drug: mechanisms of action and targets of biological pathways. Molecular Biology Report 2014, 41, 4865-4879.

8. Karplus, M.; McCammon, J. Molecular dynamics simulations of biomolecules. Nature Struct. Biol. 2002, 265, 654-652.

9. Luan, B.; Xu, G.; Feng, M.; Cong, L.; Zhou, R. Combined Computational-Experimental Approach to Explore the Molecular Mechanism of SaCas9 with a Broadened DNA Targeting Range. J. Am. Chem. Soc. 2019, 141, 6545-6552.

10. Phillips, et.al., J. C. J. Comp. Chem. 2005, 26, 1781. 
11. Jorgensen, W. L.; Chandrasekhar, J.; Madura, J. D.; Impey, R. W.; Klein, M. L. Comparison of Simple Potential Functions for Simulating Liquid Water. J. Chem. Phys. 1983, 79, 926-935.

12. Neria, E.; Fischer, S.; Karplus, M. Simulation of Activation Free Energies in Molecular Systems. J. Chem. Phys. 1996, 105, 1902-1921.

13. Beglov, D.; Roux, B. Finite representation of an infinite bulk system: Solvent boundary potential for computer simulations. J. Chem. Phys. 1994, 100, 9050-9063.

14. Huang, J.; MacKerell Jr, A. D. CHARMM36 all-atom additive protein force field: Validation based on comparison to NMR data. J. comput. Chem. 2013, 34, 2135-2145.

15. Miyamoto, S.; Kollman, P. A. SETTLE: An Analytical Version of the SHAKE and RATTLE Algorithm for Rigid Water Molecules. J. Comp. Chem. 1992, 13, 952-962.

16. Martinetz, T.; Schulten, K. Topology Representing Networks. Neur. Netw. 1994, 7, 507-522.

17. Friesner, R. A.; Banks, J. L.; Murphy, R. B.; Halgren, T. A.; Klicic, J. J.; Mainz, D. T.; Repasky, M. P.; Knoll, E. H.; Shelley, M.; Perry, J. K. et al. Glide: a new approach for rapid, accurate docking and scoring. 1. Method and assessment of docking accuracy. J. Med. Chem. 2004, 47, 1739-1749.

18. Jones, G.; Willett, P.; Glen, R. C.; Leach, A. R.; Taylor, R. Development and validation of a genetic algorithm for flexible docking. J. Mol. Biol. 1997, 267, 727-748.

19. Corbeil, C. R.; Williams, C. I.; Labute, P. Variability in docking success rates due to dataset preparation. J. Comput. Aided Mol. Des. 2012, 26, 775-786.

20. Ruiz-Carmona, S.; Alvarez-Garcia, D.; Foloppe, N.; Garmendia-Doval, A. B.; Juhos, S.; Schmidtke, P.; Barril, X.; Hubbard, R. E.; Morley, S. D. rDock: a fast, versatile and 
open source program for docking ligands to proteins and nucleic acids. PLoS Comput. Biol. 2014, 10, e1003571.

21. Trott, O.; Olson, A. J. AutoDock Vina: improving the speed and accuracy of docking with a new scoring function, efficient optimization, and multithreading. J. Comput. Chem. 2010, 31, 455-461.

22. Morris, G. M.; Huey, R.; Lindstrom, W.; Sanner, M. F.; Belew, R. K.; Goodsell, D. S.; Olson, A. J. AutoDock4 and AutoDockTools4: Automated docking with selective receptor flexibility. J. Comput. Chem. 2009, 30, 2785-2791.

23. Wang, Z.; Sun, H.; Yao, X.; Li, D.; Xu, L.; Li, Y.; Tian, S.; Hou, T. Comprehensive evaluation of ten docking programs on a diverse set of protein-ligand complexes: the prediction accuracy of sampling power and scoring power. Phys. Chem. Chem. Phys. 2016, 18, 12964-12975.

24. Wang, M.; Cao, R.; Zhang, L.; Yang, X.; Liu, J.; Xu, M.; Shi, Z.; Hu, Z.; Zhong, W.; Xiao, G. Remdesivir and chloroquine effectively inhibit the recently emerged novel coronavirus (2019-nCoV) in vitro. Cell Research 2020, 30, 269-271.

25. Holshue, M. L.; DeBolt, C.; Lindquist, S.; Lofy, K. H.; Wiesman, J.; Bruce, H.; Spitters, C.; Ericson, K.; Wilkerson, S.; Tural, A. et al. First case of 2019 novel coronavirus in the United States. New Eng. J. Med. 2020,

26. Hewlings, S. J.; Kalman, D. S. Curcumin: a review of its' effects on human health. Foods $\mathbf{2 0 1 7}, 6,92$.

27. Prasad, S.; Tyagi, A. K.; Aggarwal, B. B. Recent developments in delivery, bioavailability, absorption and metabolism of curcumin: the golden pigment from golden spice. Cancer research and treatment: official journal of Korean Cancer Association 2014, 46, 2. 
28. Zhang, W.; Du, R.-H.; Li, B.; Zheng, X.-S.; Yang, X.-L.; Hu, B.; Wang, Y.-Y.; Xiao, G.F.; Yan, B.; Shi, Z.-L. et al. Molecular and serological investigation of 2019-nCoV infected patients: implication of multiple shedding routes. Emerging microbes $\&$ infections 2020, 9, 386-389.

29. Wang, W.; Xu, Y.; Gao, R.; Lu, R.; Han, K.; Wu, G.; Tan, W. Detection of SARS-CoV-2 in Different Types of Clinical Specimens. Jama 2020,

30. Wohlleber, D.; Knolle, P. A. Infection, Immune Homeostasis and Immune Privilege; Springer, 2012; pp 93-106.

31. Neil, J. A.; Cadwell, K. The intestinal virome and immunity. The Journal of Immunology 2018, 201, 1615-1624.

32. Kaşıkcı, M. B.; Bağdathığlu, N. Bioavailability of quercetin. Current research in nutrition and food science journal 2016, 4, 146-151.

33. Yamamoto, N.; Yang, R.; Yoshinaka, Y.; Amari, S.; Nakano, T.; Cinatl, J.; Rabenau, H.; Doerr, H. W.; Hunsmann, G.; Otaka, A. et al. HIV protease inhibitor nelfinavir inhibits replication of SARS-associated coronavirus. Biochemical and biophysical research communications 2004, 318, 719-725.

34. Cao, B.; Wang, Y.; Wen, D.; Liu, W.; Wang, J.; Fan, G.; Ruan, L.; Song, B.; Cai, Y.; Wei, M. et al. A trial of lopinavir-ritonavir in adults hospitalized with severe Covid-19. New Eng. J. Med. 2020,

35. Lu, H. Drug treatment options for the 2019-new coronavirus (2019-nCoV). BioScience Trends 2020, advpub.

36. Zhang, J.; Wang, W.; Peng, B.; Peng, W.; Zhang, Y.; Wang, Y.; Wan, Y.; Chang, J.; Mao, L.; Miao, X. et al. Potential of Arbidol for Post-exposure Prophylaxis of COVID-19 Transmission. Chinaxiv 2020, 
37. Yang, Y.; Shen, H.; Zhaoshan, M.; Wang, D.; Wang, H. Potential mechanism of bromhexine in the treatment of COVID-2019. Chinese Pharmaceutical Journal 2020,

38. Li, M.; Wang, J. Prospect of ambroxol in the treatment of COVID-2019. The Chinese Journal of Clinical Pharmacology 2020,

39. Yao, X.; Ye, F.; Zhang, M.; Cui, C.; Huang, B.; Niu, P.; Liu, X.; Zhao, L.; Dong, E.; Song, C. et al. In vitro antiviral activity and projection of optimized dosing design of hydroxychloroquine for the treatment of severe acute respiratory syndrome coronavirus 2 (SARS-CoV-2). Clinical Infectious Diseases 2020,

40. Al-Bari, M. A. A. Targeting endosomal acidification by chloroquine analogs as a promising strategy for the treatment of emerging viral diseases. Pharmacology research $\& 3$ perspectives 2017,5 .

41. Hoffmann, M.; Kleine-Weber, H.; Schroeder, S.; Krüger, N.; Herrler, T.; Erichsen, S.; Schiergens, T. S.; Herrler, G.; Wu, N.-H.; Nitsche, A. et al. SARS-CoV-2 cell entry depends on ACE2 and TMPRSS2 and is blocked by a clinically proven protease inhibitor. Cell 2020,

42. McChesney, E. W.; Banks Jr, W. F.; Fabian, R. J. Tissue distribution of chloroquine, hydroxychloroquine, and desethylchloroquine in the rat. Toxicology and applied pharmacology 1967, 10, 501-513.

43. Liu, X.; Li, Z.; Liu, S.; Chen, Z.; Zhao, Z.; Huang, Y.-y.; Zhang, Q.; Wang, J.; Shi, Y.; $\mathrm{Xu}, \mathrm{Y}$. et al. Therapeutic effects of dipyridamole on COVID-19 patients with coagulation dysfunction. MedRxiv 2020,

44. Zoete, V.; Cuendet, M. A.; Grosdidier, A.; Michielin, O. SwissParam: a fast force field generation tool for small organic molecules. J. Comput. Chem. 2011, 32, 2359-2368. 